\title{
28 Research Suare \\ Dietary Restriction Induces a Stable Metabolic Obesity Phenotype in Drosophila Melanogaster
}

\section{Oscar Hilary Asiimwe}

Kampala International University - Western Campus

Sheu Oluwadare Sulaiman

Kampala International University - Western Campus

Juma John Ochieng

Kampala International University - Western Campus

David Onanyang

Gulu University

\section{Lawrence Obado Osuwat}

SUN: Soroti University

John Mark Ndinawe

Kampala International University - Western Campus

Alfred Omachonu Okpanachi

Kampala International University - Western Campus

Keneth Iceland Kasozi ( $\nabla$ kicelandy@gmail.com )

Kampala International University - Western Campus https://orcid.org/0000-0002-5763-7964

\section{Research note}

Keywords: Metabolic obesity, Restrictive diets, Obesity, triglyceride, catalase, Drosophila melanogaster

Posted Date: June 25th, 2021

DOI: https://doi.org/10.21203/rs.3.rs-645996/v1

License: (c) (i) This work is licensed under a Creative Commons Attribution 4.0 International License. Read Full License 


\section{Abstract}

\section{Objective}

Challenges associated with current nutritional models to induce obesity in Drosophila melanogaster created a rationale for this study. The objective of the study was to investigate biochemical changes associated with high-fat diet (HFD), high sucrose diet (HSD), and a protein-restricted diet (DR) to induce a healthy metabolic obesity state. Drosophila melanogaster were fed to four experimental diets: regular food (control), HFD, HSD, and DR, for four weeks. Peristaltic waves were measured on $3^{\text {rd }}$ instar larvae, while negative geotaxis, body mass, catalase activity; and total triglycerides, sterol, and protein were measured in adult Drosophila melanogaster.

\section{Results}

DR produced a Drosophila melanogaster phenotype which had superior adaptive advantages than that generated from HFD and HSD. HFD was the best phenotype during larval stages; however, locomotory, body mass, triglyceride, sterol concentrations, and catalase activity were highest in the DR phenotype during adulthood. High catalase activity and high triglyceride content demonstrated a balanced and healthy metabolic obesity status than in other phenotypes in the adult stage. Evolutionary changes are responsible for the selective advantage of the DR phenotype over the HFD phenotype. Prospective studies to guide therapy and community behavior should place more emphasis on the DR phenotypes in Drosophila melanogaster.

\section{Introduction}

Drosophila melanogaster (Drosophila) animal disease model is increasingly being used because of the high cost of maintaining mammalian models (Coogan, 2013). Drosophila accumulates lipids in both the fat and non-fat tissue in a dose-dependent manner in a shorter period, unlike mice which take months to become obese (1). Most of the major metabolic enzymes in mammals are conserved in Drosophila, and the genes that regulate lipid metabolism are well conserved (2). Lipids in Drosophila are stored as triglycerides in the fat tissue, similar to triglyceride storage in mammals' adipose tissue $(3,4)$. Additionally, the molecular mechanism that controls the metabolism of neutral lipids in cellular lipid droplets (lipophorins) resembles that in mammalian pathways of lipoproteins (5). Like other insects when fed a high-fat diet (HFD), Drosophila stores other forms of sterols such as ergosterol, stigmasterol, zymosterol, and campesterol depending on the dietary fat source and the sterol requirements $(6,7)$. The excess sterols from dietary sources are interconverted to triglycerides as the main circulating and storage lipid energy reserves depending on the energy balance and metabolic requirements for specific body processes (8).

Drosophila has proved to be a great model of obesity $(9,10)$; however, studies on diet-induced phenotypes are scarce. For example, dietary protein restriction has been associated with increased lifespan in adult and larval stages of Drosophila (11). Furthermore, dietary restriction has been associated with increased expression of antioxidant genes, increasing stress resistance and locomotory activity (12). In Drosophila 
larvae, restricted nutrition has been associated with higher fat reserves in adult flies due to increased expression of fat mobilizing genes and lipid storage droplet-2 in mature flies (13). However, effects of coconut and sucrose as feed additives on the Drosophila phenotype have not been explored. This study's objective was to assess effects of high fat and sucrose diets on larval and adult fitness, mass, sterol and triglyceride content in Drosophila melanogaster.

\subsection{Methods}

\subsection{Laboratory animals}

The study was done using Yellow white strain $w^{1118}$ of Drosophila Melanogaster obtained originally from the National Species Stock Center (Bowling Green, OH, USA). Roughly 1200 adult flies (with equal numbers of females and males were mated and maintained for two generations at the Institute of Biomedical Research of Kampala International University Western Campus, Uganda. The flies were divided into four groups exposed to regular food (control), high-fat diet (HFD), high sucrose diet (HSD), 15\% protein-restricted diet ( $D R=15 \%$ less yeast than in regular feed) for two weeks under $70 \%$ humidity, $24-26^{\circ} \mathrm{C}$ temperature and 12:12 $\mathrm{h}$ light/dark cycles. Flies at the second generation, which were age-matched flies, were transferred and placed in $500 \mathrm{ml}$ plastic flasks containing $25 \mathrm{ml}$ of fly food with extra yeast added and left there for 15 $\mathrm{h}$ at $25^{\circ} \mathrm{C}$. Eggs laid on the food surface and the container wall were removed by using a tender brush and left on the food to grow in the incubator (14). First instar larvae were collected within 2-3 hours of egg hatching. Thirty third instar larvae were collected into fresh vials on day three, placed on new experimental specific media and allowed to grow to adulthood.

\subsection{Experimental design}

Drosophila regular food contained cornmeal $7 \% \mathrm{w} / \mathrm{v}$, dextrose $7.5 \% \mathrm{w} / \mathrm{v}$, yeast $1.5 \% \mathrm{w} / \mathrm{v}$, nipagin $2.33 \% \mathrm{v} / \mathrm{v}$, agar base $1.05 \% \mathrm{w} / \mathrm{v}$, propionic acid $0.37 \% \mathrm{w} / \mathrm{v}$ in a liter of food (control group). HFD was prepared by adding $10 \% \mathrm{w} / \mathrm{v}$ food-grade coconut oil to the regular cornmeal food (15). HSD was prepared by adding $150 \mathrm{mM}$ sugar to regular cornmeal food $(10,15)$. DR was prepared by reducing the yeast composition by $15 \%$ (11). The larval media contained the same feed composition at a concentration of $50 \%$ except for agar and nipagin. Each group contained ten third instar larvae. Subsequently, ten adults were kept in each vial and experiments were conducted in triplicates for four weeks from the date of egg collection (larval experiments for one day while adults emerged after day 10).

\subsection{Complete peristaltic waves of third instar larvae}

This was performed on $1 \%$ agar in distilled water on Petri dishes to increase visibility and ease recording of movements (16). Each larva was washed in distilled water to clean them of any food and transferred using a smooth brush to a fresh plate. The larvae were allowed to acclimatize for 1 minute, and video recordings were conducted from the top. The number of peristaltic waves per minute was counted in 3 consecutive trials.

\subsection{Negative geotaxis assay and locomotor activity assays}


After two weeks, negative geotaxis was investigated as previously described (17) with minor modifications. Ten flies from the respective groups were immobilized under light anesthesia with ice. After 10 minutes of recovery, the flies were gently tapped to the bottom of the column, and the time they took to reach the height of $8 \mathrm{~cm}$ was recorded. The tests were repeated three times for each group at one-minute intervals.

\subsection{Fly Body mass}

Each fly was frozen, and its body weight measured using a Sartorius microbalance (18). Whole fly samples were then homogenized in $100 \mu \mathrm{l}$ of cold $0.05 \%$ phosphate-buffered saline tween solution, and the homogenate centrifuged at $13,000 \mathrm{rpm}$ for 3 minutes (19). The supernatant was immediately stored at $4^{0} \mathrm{C}$ for biochemical analysis.

\subsection{Determination of total triglycerides and sterol concentrations from the supernatant}

Total triglyceride and sterol levels were measured using Cypress Diagnostics triglyceride and cholesterol oxidase kits, respectively. A commercial coupled colorimetric assay (CCA) protocol was used to indirectly measure the triglyceride in the form of a quinone imine dye at $540 \mathrm{~nm}$ absorbance (20). A fluorometric assay protocol was used to indirectly measure the sterol in the form of resorufin at $590 \mathrm{~nm}$ fluorescence (19).

\subsection{Determination of total protein and catalase activity from the supernatant}

The total protein was indirectly measured using Cypress Diagnostics kit and a Bradford assay protocol (17).

Catalase activity was determined by following a protocol developed by (21) and customized by (17). A calibration curve was generated in the form $y=m x+c$ using standard catalase concentrations for which the corresponding foam heights were determined with the defined unit of catalase activity. $100 \mu \mathrm{l}$ of catalase solution was pipetted in $13 \mathrm{~mm}$ diameter $\times 100 \mathrm{~mm}$ height test tubes, $100 \mu$ of $1 \%$ Triton X-100 and $100 \mu$ l of undiluted hydrogen peroxide (30\%) were added to the solutions, mixed thoroughly, and incubated at room temperature. After reaction completion, the height of $\mathrm{O}_{2}$-forming foam that remained constant for 15 minutes in the test tube was measured using a ruler. The corresponding catalase activity was ascertained and expressed as $\mathrm{mg} / \mathrm{ml}$ of protein. A standard curve was constructed from which an equation was generated in the form $\mathrm{y}=\mathrm{mx}+\mathrm{c}$ i.e., Absorbance $(\mathrm{y})=0.0432$ concentration $(\mathrm{x})+0.013$.; $R^{2}=$ 0.9973 . The above experimental protocol and the standard curve generated were used to determine catalase activity for HFD, HSD, and yeast supplemented base food fed fly samples.

\subsection{Statistical analysis}

Graphpad prism version 6 software (GraphPad Software, La Jolla, CA, USA) was used for statistical analysis and the results were reported as mean \pm SEM. A Tukey's test was used for inferential statistics, and $\mathrm{p}<0.05$ was considered to represent significance. 


\subsection{Results}

\subsection{Restrictive diets led to better phenotypes in Drosophila melanogaster}

In the larval stages, DR was associated with significantly low peristaltic movements compared to HFD (Fig. 1A). In adults, DR was associated with increased body mass (Fig. 1B) and significantly increased locomotory activity (Fig. 1C). Also, HSD was associated with high peristaltic movements than the control feed in the larval stages, although no significant differences were observed between HSD and the control on body mass and locomotion (Table 1).

Table 1

Tukey's multiple comparison test on peristaltic waves in 3rd instar larvae, body mass, negative geotaxis, triglycerides, sterols, and total protein and catalase activity in adult male Drosophila melanogaster.

\begin{tabular}{|c|c|c|c|c|c|c|c|c|}
\hline \multirow{2}{*}{$\begin{array}{l}\text { Tukey's } \\
\text { multiple } \\
\text { comparisons } \\
\text { test }\end{array}$} & $\mathbf{N}$ & $\begin{array}{l}\text { Complete } \\
\text { peristatic } \\
\text { waves }\end{array}$ & Mass & $\begin{array}{l}\text { Negative } \\
\text { geotaxis }\end{array}$ & Triglycerides & sterols & $\begin{array}{l}\text { Total } \\
\text { protein }\end{array}$ & Catalase \\
\hline & & \multicolumn{7}{|c|}{ Adjusted P Value } \\
\hline $\begin{array}{l}\text { Control vs. } \\
\text { DR }\end{array}$ & 60 & 0.0236 & $\begin{array}{l}< \\
0.0001\end{array}$ & 0.0002 & $<0.0001$ & $\begin{array}{l}< \\
0.0001\end{array}$ & $\begin{array}{l}< \\
0.0001\end{array}$ & 0.0003 \\
\hline $\begin{array}{l}\text { Control vs. } \\
\text { HSD }\end{array}$ & 60 & $<0.0001$ & 0.8452 & 0.7538 & $<0.0001$ & $\begin{array}{l}< \\
0.0001\end{array}$ & $\stackrel{<}{<.0001}$ & 0.0262 \\
\hline $\begin{array}{l}\text { Control vs. } \\
\text { HFD }\end{array}$ & 60 & $<0.0001$ & 0.1442 & 0.2641 & $<0.0001$ & 0.0002 & $\dot{0} 0001$ & 0.0416 \\
\hline DR vs. HSD & 60 & $<0.0001$ & $\begin{array}{l}< \\
0.0001\end{array}$ & 0.0005 & $<0.0001$ & $\begin{array}{l}< \\
0.0001\end{array}$ & $\stackrel{<}{<.0001}$ & 0.0167 \\
\hline DR vs. HFD & 60 & $<0.0001$ & $\begin{array}{l}< \\
0.0001\end{array}$ & $<0.0001$ & $<0.0001$ & $\begin{array}{l}< \\
0.0001\end{array}$ & 0.9988 & 0.0107 \\
\hline HSD vs. HFD & 60 & $<0.0001$ & 0.4141 & 0.0665 & $<0.0001$ & $\begin{array}{l}< \\
0.0001\end{array}$ & $\begin{array}{l}< \\
0.0001\end{array}$ & 0.9863 \\
\hline
\end{tabular}

\subsection{Protein restrictive diet improved fat storage and antioxidant enzymes}

In adult stages, DR was associated with significantly increased triglycerides (Fig. 2A), sterols (Fig. 2B), low total protein (Fig. 2C), and high catalase activity (Fig. 2D). Furthermore, HFD had high triglycerides and sterol levels only lower than those in the DR; however, HFD had the highest total protein levels and significantly comparable levels of total protein and catalase to HSF (Table 1).

\section{Discussion}


The study shows that protein-restricted dietary nutrition results in superlative phenotypes than high-fat diets. The study also shows that stress experienced during early developmental stages in life (larvae) could lead to reduced activity due to reduced impulse transduction (16). However, this favors the development of adaptive changes with increased foraging for food, resulting in an increased body mass, demonstrating evolutionary changes that would favor survival during adulthood (11). Furthermore, the study findings are similar to those of previous studies where diet restriction improved the ability to resist stress (13), increased mitochondrial density and respiratory activity, enhanced fat metabolism and physical activity (22). Besides, high fat diet-induced obesity showed $a \geq 50 \%$ reduction in Drosophila's competitive ability $(10,23)$. On the other hand, overnutrition during childhood would lead to the development of laziness and reluctance to forage for food, and this would justify the decreased activity in adults exposed to a lot of food in childhood. DR-induced obesity modelling in Drosophila did not affect the nervous coordination potential of obese flies. Thus, it caused higher competitive ability, unlike HFD- and HSD-induced obesity models that negatively affected nervous coordination ability, causing lesser competitive ability. These results are supported by previous studies, which reported competitive ability under obese conditions is independent of the level and degree of adiposity (24). Findings in this study demonstrate the reliability of Drosophila as a research model to study metabolic and evolutionary conditions in humans $(9,10)$.

We also showed that DR in adulthood caused increased triglycerides, sterols and high catalase activity. These findings demonstrate the improved metabolic state in the Drosophila phenotype. Triglycerides provide energy, and these are markers of obesity in Drosophila (8). Increased energy storage versus expenditure is associated with improved antioxidant balance, which is important to control reactive oxygen species arising from oxidation of lipids and oxidative stress $(25,26)$. The major storage forms of sterols in Drosophila is dehydrocholesterol and ergosterol (19). The DR phenotype had the highest levels of sterols than the HFD and HSD fed obese flies, and mechanisms for these differences remain to be explored.

Total protein levels were highest in HSD, providing evidence that HSD-induced obesity in Drosophila could face major increased protein levels compared to HFD. This is because HFD has been associated with increased lipid peroxidation, which causes increased tissue death and reduced lifespan in Drosophila $(27,28)$. Furthermore, HSDs are characterized by a hypertonicity-a condition that hastens autolysis $(29)$. On the other hand, challenges observed with HFD and HSD models help identify them as better cancer research models (30). High catalase activity demonstrates the superlative advantage associated with DR for the induction of obesity in Drosophila. Increased endogenous catalase enzyme activity serves as a natural defense mechanism against oxidative stress in pathological conditions such as obesity (31). Our findings are similar to previous studies that reported DR association with increased mitochondrial density and respiratory activity, causing elevated antioxidant enzyme activity. This is unlike the HFD and HSD fed obese flies which were associated with high levels of oxidative stress and tissue death $(15,22)$. Furthermore, sugar diets at either low or high supplementation alter physiological function (reproduction) due to body size changes and organ size (32).

In this study, induction of obesity by DR produced a competitively more active and obese fly phenotype compared to the HFD and HSD fed obese phenotypes. The study's findings demonstrate a need to explore 
more active and appropriate models for modeling obesity, emphasizing evolutionary adaptive changes to promote knowledge on healthy metabolic obesity.

\section{Limitations}

Molecular markers describing the metabolism of triglycerides were not investigated in this study due to financial and infrastructural challenges. Further studies would emphasize the crosstalk between a balanced diet and obesity to generate knowledge to address metabolic obesity. This would enhance further understanding of the immunological modulators of inflammation, second messengers and modulators of gene expression that can lead to the development of novel therapeutical options and healthy lifestyles.

\section{Abbreviations}

HFD High-fat diet HSD High sucrose diet DR Restrictive diet w/v Weight by volume v/v Volume by volume

\section{Declarations}

\section{Ethical approval and consent to participate}

Ethical considerations were followed. This research model was selected based on following the replacement protocol for ethics in animal research and registered under number Nr.UG-REC-023/201916. Consent to participate was not applicable.

\section{Consent to publish}

Not applicable

\section{Competing interests}

The authors declare no conflicts of interest exist.

\section{Author contributions}

$\mathrm{OHA}$ and KIK conceptualized and designed the study, $\mathrm{OHA}$ and KIK conducted data acquisition and analysis while SSO, JJO, DO, JMN, LOO, AOO interpreted the data. KIK and OHA drafted the manuscript. All authors revised it critically for intellectual content, gave final approval for publication and all authors remain in agreement to ensure that questions related to the work are appropriately resolved.

\section{Availability of data and materials}




\section{Funding statement}

Not applicable

\section{Acknowledgements}

The authors wish to thank Dr. Marta Vicente-Crespo for her technical support and Sayansi IxD and DrosAfrica that supported the Institute of Biomedical Research establishment in KIU with materials, equipment, and training workshops.

\section{References}

1. Birse RT, Choi J, Reardon K, Rodriguez J, Graham S, Diop S, et al. High-Fat-Diet-Induced Obesity and Heart Dysfunction Are Regulated by the TOR Pathway in Drosophila. Cell Metab. 2010;12(5):533-44.

2. Owusu-ansah E, Perrimon N. Modeling metabolic homeostasis and nutrient sensing in Drosophila: Implications for aging and metabolic diseases. J Dis Model Mech. 2014;7(3):343-50.

3. Bharucha KN, Tarr P, Zipursky SL. A glucagon-like endocrine pathway in Drosophila modulates both lipid and carbohydrate homeostasis. Am J Physiol- Endocrinol Metab. 2008;5(2008):3103-10.

4. Diangelo JR, Birnbaum MJ. Regulation of Fat Cell Mass by Insulin in Drosophila melanogaster. J Mol Cell Biol. 2009;29(24):6341-52.

5. Palm W, Sampaio JL, Brankatschk M, Carvalho M, Mahmoud A, Shevchenko A, et al. Lipoproteins in Drosophila melanogaster - Assembly, Function, and Influence on Tissue Lipid Composition. PLOS Genet. 2012;8(7).

6. Carvalho M, Sampaio JL, Palm W, Brankatschk M, Eaton S, Shevchenko A. Effects of diet and development on the Drosophila lipidome. Mol Syst Biol. 2012;8(600):1-17.

7. Liu Z, Huang X. Lipid metabolism in Drosophila: development and disease Using Drosophila System to Study Lipid Metabolism Lipids Function in Drosophila Early Development. Acta Biochim Biophys Sin ABBS. 2013;45(1):44-50.

8. Heier C, Kühnlein RP. Triacylglycerol Metabolism in Drosophila melanogaster. Genetics. 2018;210(December):1163-84.

9. Trinh I, Boulianne GL. Modeling Obesity and Its Associated Disorders in Drosophila. Physiology. 2013;28(2):117-24.

10. Villanueva JE, Livelo C, Trujillo AS, Chandran S, Woodworth B, Andrade L, et al. Time-restricted feeding restores muscle function in Drosophila models of obesity and circadian-rhythm disruption. Nat Commun. 2019;10(2019):1-17.

11. Krittika S, Lenka A, Yadav P. Evidence of dietary protein restriction regulating pupation height, development time and lifespan in Drosophila melanogaster. Biol Open. 2019. 
12. Ghimire S, Kim MS. Enhanced Locomotor Activity Is Required to Exert Dietary Restriction-Dependent Increase of Stress Resistance in Drosophila. Oxid Med Cell Longev [Internet]. 2015;2015:1-8. Available from: http://www.hindawi.com/journals/omcl/2015/813801/.

13. Rehman N, Varghese J. Larval nutrition influences adult fat stores and starvation resistance in Drosophila. PLoS One. 2021.

14. Schou MF. Fast egg collection method greatly improves randomness of egg sampling in Drosophila melanogaster. Fly (Austin). 2013.

15. Paula MT, De, Rósula M, Silva P, Araujo SM, Bortolotto VC, Meichtry LB, et al. High-Fat Diet Induces Oxidative Stress and MPK2 and HSP83 Gene Expression in Drosophila melanogaster. J Oxidative Med Cell Longev. 2016;2016(4018157):1-12.

16. Gjorgjieva J, Berni J, Evers JF, Eglen SJ. Neural circuits for peristaltic wave propagation in crawling Drosophila larvae: analysis and modeling. Front Comput Neurosci [Internet]. 2013;7:24. Available from: http://journal.frontiersin.org/article/10.3389/fncom.2013.00024/abstract.

17. Kasozi KI, Bukenya A, Eze ED, Kasolo J, Tayebwa DS, Ssempijja F, et al. Low concentrations of Lactobacillus rhamnosus GG (Yoba ${ }^{\circledR}$ ) are safe in male Drosophila melanogaster. BMC Res Notes. 2019 May 14;12(1).

18. Ma J, Benson AK, Kachman SD, Jacobsen DJ, Harshman LG. Drosophila melanogaster Selection for Survival after Infection with Bacillus cereus Spores: Evolutionary Genetic and Phenotypic Investigations of Respiration and Movement. Int J Evol Biol [Internet]. 2013 Mar 21;2013:1-12. Available from: https://www.hindawi.com/journals/ijeb/2013/576452/.

19. Tennessen JM, Barry WE, Cox J, Thummel CS. Methods for studying metabolism in Drosophila. Methods. 2014;68(1):105-15.

20. Hildebrandt A, Bickmeyer I, Kühnlein RP. Reliable Drosophila body fat quantification by a coupled colorimetric assay. PLoS One. 2011;6(9):1-6.

21. Iwase T, Tajima A, Sugimoto S, Okuda K, Hironaka I, Kamata Y, et al. A Simple Assay for Measuring Catalase Activity: A Visual Approach. Sci Rep. 2013;3(1):1-4.

22. Kapahi P, Kaeberlein M, Hansen M, Jolla L. Dietary restriction and lifespan: Lessons from invertebrate models. Aging Res Rev. 2018;39(2017):3-14.

23. Heinrichsen ET, Zhang H, Robinson JE, Ngo J, Diop S, Bodmer R, et al. Metabolic and transcriptional response to a high-fat diet in Drosophila melanogaster. Mol Metab. 2014;3(1):42-54.

24. Brookheart RT, Michel Cl, Schaffer JE. As A Matter of Fat. Cell Metab. 2010;10(1):9-12.

25. Katewa SD, Akagi K, Bose N, Rakshit K, Brem RB, Ramanathan A, et al. Peripheral circadian clocks mediate dietary restriction dependent changes in lifespan and fat metabolism in Drosophila. Cell Metab. 2017;23(1):143-54.

26. Morris SNS, Coogan C, Chamseddin K, Fernandez-Kim SO, Kolli S, Keller JN, et al. Development of dietinduced insulin resistance in adult Drosophila melanogaster. Biochim Biophys Acta - Mol Basis Dis. 2012;1822(8):1230-7. 
27. Trindade de Paula M, Poetini Silva MR, Machado Araujo S, Cardoso Bortolotto V, Barreto Meichtry L, Zemolin APP, et al. High-Fat Diet Induces Oxidative Stress and MPK2 and HSP83 Gene Expression in Drosophila melanogaster. Oxid Med Cell Longev. 2016.

28. Skorupa DA, Dervisefendic A, Zwiener J, Pletcher SD. Dietary composition specifies consumption, obesity, and lifespan in Drosophila melanogaster. Aging Cell. 2008;7(2008):478-90.

29. Na J, Sweetwyne MT, Seo A, Park D, Susztak K, Ross L, et al. Diet-Induced Podocyte Dysfunction in Drosophila and Mammals Article Diet-Induced Podocyte Dysfunction in Drosophila and Mammals. CellReports. 2015;12(4):636-47.

30. Hirabayashi S. The interplay between obesity and cancer: a fly view. Dis Model Mech. 2016;9(2016):917-26.

31. Amoako S, Yahaya A, Sarfo JK. Catalase activity of cassava (Manihot esculenta) plant under African cassava mosaic virus infection in Cape coast, Ghana. African J Biotechnol. 2015;14(14):1201-6.

32. Klepsatel P, Knoblochová D, Girish TN, Dircksen H, Gáliková M. The influence of developmental diet on reproduction and metabolism in Drosophila. BMC Evol Biol. 2020.

\section{Figures}
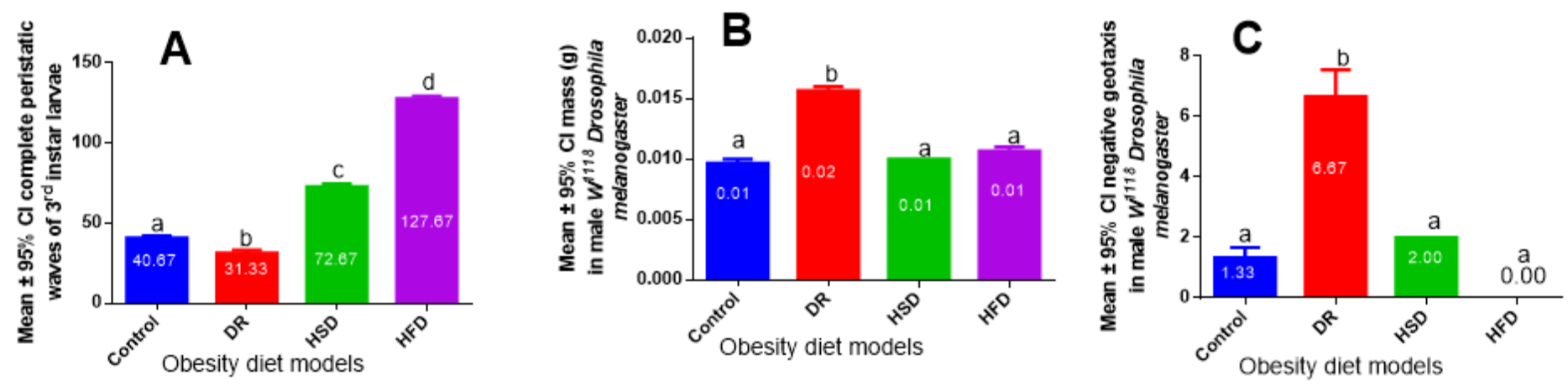

Figure 1

Changes in Drosophila melanogaster after induction of obesity (A) Complete peristaltic waves of third instar larvae (B) Mass and (C) Negative geotaxis. 

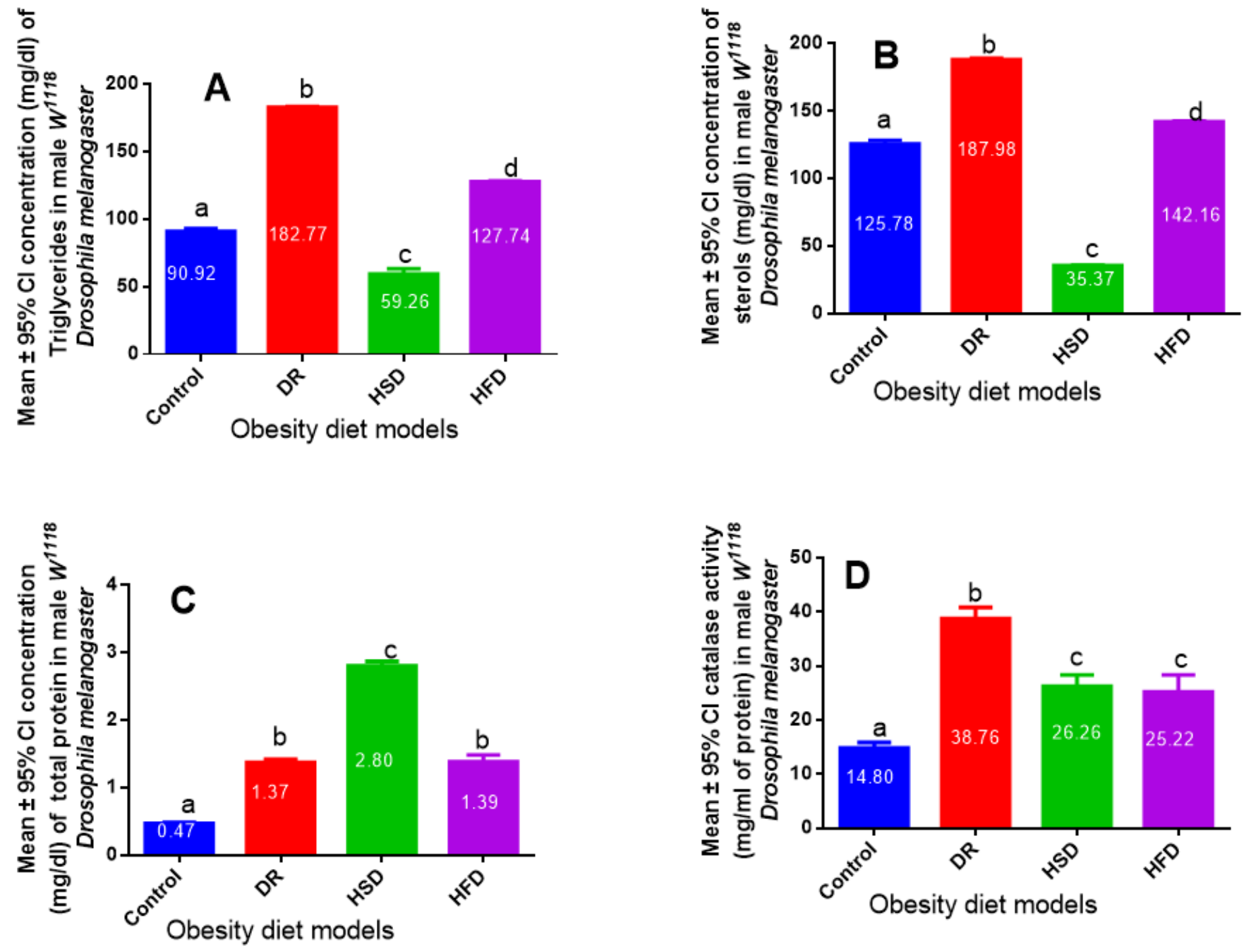

Figure 2

Changes in Drosophila melanogaster after induction of obesity (A) Triglyceride (B) sterols (C) Total protein (D) Catalase activity. Different superscripts indicate significant differences in experimental groups. 